\title{
Pay-load estimation of a 2DOF flexible link robot using a delta-operator technique
}

\author{
Jensen, Morten Rostgaard; Poulsen, Niels Kjølstad; Ravn, Ole
}

Published in:

Proceedings of the 2001 IEEE International Conference on Control Applications

Link to article, DOI:

10.1109/CCA.2001.973872

Publication date:

2001

Document Version

Publisher's PDF, also known as Version of record

Link back to DTU Orbit

Citation (APA):

Jensen, M. R., Poulsen, N. K., \& Ravn, O. (2001). Pay-load estimation of a 2DOF flexible link robot using a delta-operator technique. In Proceedings of the 2001 IEEE International Conference on Control Applications (pp. 248-253). https://doi.org/10.1109/CCA.2001.973872

\section{General rights}

Copyright and moral rights for the publications made accessible in the public portal are retained by the authors and/or other copyright owners and it is a condition of accessing publications that users recognise and abide by the legal requirements associated with these rights.

- Users may download and print one copy of any publication from the public portal for the purpose of private study or research.

- You may not further distribute the material or use it for any profit-making activity or commercial gain

- You may freely distribute the URL identifying the publication in the public portal 


\title{
Pay-Load Estimation of a 2DOF Flexible Link Robot using a Delta-Operator Technique
}

\author{
M. Rostgaard ${ }^{\star}$, N.K. Poulsen ${ }^{\star}$, O. Ravn ${ }^{\diamond}$ \\ *Department of Mathematical Modelling, Building 321, Richard Petersens Plads, \\ Technical University of Denmark, DK-2800 Kgs. Lyngby, Denmark. \\ mrj, nkp@imm.dtu.dk www.imm.dtu.dk/ nkp \\ -Department of Automation, Building 326, Elektrovej \\ Technical University of Denmark, DK-2800 Kgs. Lyngby, Denmark. \\ or@iau.dtu.dk www.iau.dtu.dk/ or
}

\begin{abstract}
The paper presents a new method for online identification of pay-loads for a twolink flexible robot. The method benefits from the close correspondence between parameters of a discrete-time model represented by means of the Delta-Operator, and those of the underlying continuous-time model. Although the applied principle might be general in nature, the paper is applied to the well-known problem of identifying a pay-load of a moving flexible robot. This problem is almost impossible to solve by measurements, so an estimation technique must be applied. The presented method benefits from the close correspondence with the continuoustime representation to allow a scalar and implicit adaptive technique which based on flexibility measurements leads to the online estimation of the pay-load.
\end{abstract}

Keywords : Flexible Link Robot; DeltaOperator; System Identification; Parameter Estimation; Adaptive Control.

\section{INTRODUCTION}

Flexible robot systems are motivated by a desire for better arm-weight to pay-load ratios, shorter travel-times and a lower energy consumption. The desire for high-performance manipulators and the benefits offered by a lightweight flexible link capable of maneuvering large pay-loads have lead to analysis of the behavior of the dynamics in which flexibility is the essential issue. The high-performance requirements will inevitably produce designs that during operation will excite vibrations in the manipulator structure.

The flexibility generates a severe problem in controlling the motion due to the inevitably excitation of structural vibrations which affect the accuracy of the manipulator. Therefore a successful controller implementation of a flexi- ble manipulator system is contingent on achieving acceptable performance taking into account variations in e.g. pay-load and environmental disturbances.

The aim of the controller is to suppress the structural vibration while in addition to minimize the cycle time of the manipulator system. For flexible manipulator systems, it is necessary to use a model-based controller in order to mitigate the first harmonics. However, changes in pay-load degrades the model and consequently the performance of the control system, unless some sort of adaptation or gain-scheduling is taken into account to estimate these effects.

In order to investigate different aspects of control of flexible links robot configurations an experimental setup has been made. A simulation model has been develloped as well and has been used for producing the results shown in this paper. This experimental setup consists of two very flexible links with two actuators located in the joints. In this work the links are moving in the horizontal plane making gravity ignorable. The geometry of the links makes the predominant bending take place in this plane making it possible to ignore torsion. The actuators are DC-motors with a sufficient gear ratio and tachometers making an analog velocity feedback feasible, this suppresses the friction and other non-linearities in the actuators. Apart from the tachometers there are two kinds of sensors on the setup, a potentiometer in each joint enabling a measurement of the position of the joint and a number of strain gauges located on each link enabling the measurement of the bending of the link. The simulation model used in this study is 
derived using physical modelling from the this setup. A photograph of the setup is shown in Figure 1 and a schematic view in Figure 2.

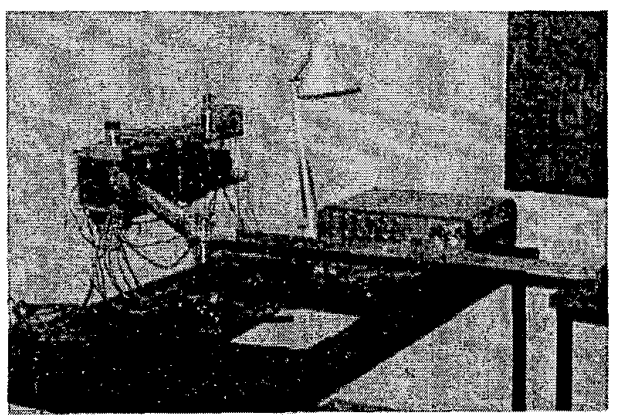

Fig. 1. The robot consists of two flexible links and two actuators (DC-motors). It is equipped with two tachometers and four strain gauges in order to measure the link angles and deflection, respectively figure

In [5] experiments are described for a flexible single-beam system, where time-varying blackbox input-output forms are proposed as suitable descriptions of the system. In [3] experimental results featuring gravity compensation are presented for a double link robot with a flexible forearm. But like other references, practical algorithms seem to ignore the fact that one could construct an adaptation technique that directly gains insight to the pay-load parameter.

This is topic for the present work. By deducting a linear state-space model describing the pay-load parameter's influence in the continuous-time model, it is possible to apply a Delta-Operator technique for estimation of this parameter in discrete-time.

To demonstrate this, the paper starts in Section II by giving the equations dscribing a two link manipulator system. In Section IV the Delta-Operator is introduced, and also the discrete-time estimation technique is presented. As the linearized model is in general sufficient to capture the dynamics of each flexible link, it is demonstrated in Section V, that the pay-load may be estimated using a recursive parameter estimation technique.

It is worth noting that the presented estimation technique is tied to the Delta-Operator. Provided the discretizing had been based on the conventional shift-operator, one would inevitably had lost the clear physical interpretation of the parameters, and the method would have failed. However, as the presented method is discretised using the Delta-Operator [4], a close correspondence appear between the

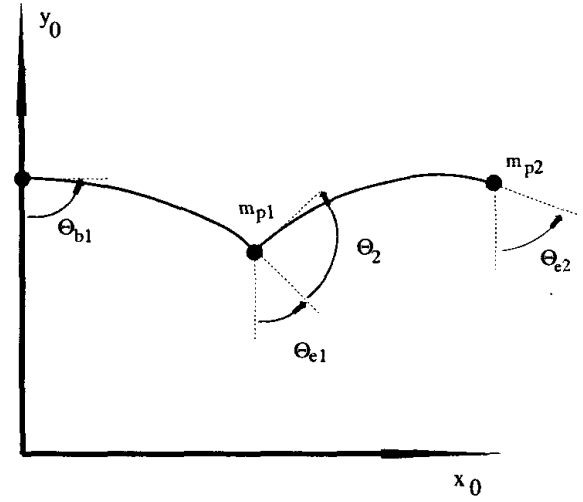

Fig. 2. The flexible robot system consists of two flexible links that are coupled together through actuator 2 figure

discrete-time parameters and the underlying continuous-time system.

\section{DESIGN MODEL OF SYSTEM}

The flexible manipulator system studied here, see Fig. 2, carries a pay-load, $m_{p}$, at its tip and moves in the horizontal plane. The active degrees of freedom are the two rotational angles $\theta_{b 1}$ and $\theta_{2}$.

In literature the equations of motions are commonly modelled by either a Finite Element Method (see. e.g. [8]) or the Eigenvalue Method (see e.g. [2]). As the latter method is normally considered more accurate when only a limited number of modes are included, cf. [1], the following description is be based on this approach, cf. [7].

The model of the flexible link robot consists of four parts; namely the models for the two actuators and the two arms. The dynamics of the flexible arms can be described by a PDE which can be transfered into a ODE by using the method of separation of variable. In that case the deflection, $w_{j}(x, t), j=1,2$, of the arms is approximated by a finite sum of contributions

$$
w_{j}(x, t)=\sum_{i=1}^{n} \varphi_{j i}(x) q_{i j}(t)
$$

where $\varphi_{j i}(x)$ and $q_{j i}(t)$ are the normal and harmonic function of mode $i$ and $\operatorname{arm} j$, respectively.

Introduce the notation:

$$
\begin{aligned}
\underline{q} & =\left[q_{11}, \ldots q_{1 n}, q_{21}, \ldots q_{2 n}\right]^{T}, \\
\underline{u} & =\left[\begin{array}{l}
u_{1} \\
u_{2}
\end{array}\right]
\end{aligned}
$$


Then the description of the flexibility can linearized and be brought into the following compact form:

$$
\underline{\ddot{q}}=M_{1} \underline{q}+M_{2} \underline{\dot{q}}+M_{3} \underline{\ddot{q}}+M_{4} \underline{u}+M_{5} \underline{\dot{u}}
$$

where the matrices, $M_{2}, M_{3}$ and $M_{5}$ are linearly dependent on $m_{p}$. Notice, the matrices depend on the linearization point. In this case the matrices depend only on $\theta_{2}$. Also, notice the angular acceleration, $q$, occurs on both side of the $\mathrm{i}$ equation.

Also the actuator dynamics can be written in a compact form

$$
\underline{\dot{\theta}}=M_{6} \underline{q}+M_{7} u
$$

where

$$
\underline{\theta}=\left[\begin{array}{c}
\theta_{b 1} \\
\theta_{2}
\end{array}\right]
$$

Now the compact description in (3) (4) is to be transformed into a state space description. The algebraic loop (related to $\underline{q}$ in (3)) can be solved if the following matrix inverse exists,

$$
\Lambda=\left(I-M_{3}\right)^{-1}
$$

Notice $M_{3}$ depend linearly on the pay load mass, $m_{p}$. Taking the states

$$
x=\left[\begin{array}{c}
\frac{\theta}{q} \\
\underline{q}-\underline{\Lambda} M_{5} u
\end{array}\right]
$$

one obtains easily the state space description

$\dot{x}=\underbrace{\left[\begin{array}{ccc}0 & M_{6} & 0 \\ 0 & 0 & I \\ 0 & \Lambda M_{1} & \Lambda M_{2}\end{array}\right]}_{\underline{A}} x+\underbrace{\left[\begin{array}{c}M_{7} \\ \Lambda M_{5} \\ \Lambda M_{2} \Lambda M_{5}+\Lambda M_{4}\end{array}\right]}_{\underline{B}} u$

where the state transition matrix is of order $2+4 n$ with $n$ as the number of considered modes. For an arbitrary linearization angle, this representation is a linear approximation to the dynamics, i.e. $M_{i}$ are functions of the linearization angle. Thus, one can use the measurements of $\theta_{2}$ to obtain a running linear description around the actual orientation.

\section{A. Structuring the model in the pay load param- eter}

Since the pay load mass is unknown or even time varying we will have rephrase the model in such a way that it is possible to estimate the pay load mass, $m_{p}$. The suggested method takes starting point in the split-up of the state transition and state control matrices of the form

$$
\underline{A}=\underline{A}^{0}+m_{p} \underline{A}^{m}, \quad \underline{B}=\underline{B}^{0}+m_{p} \underline{B}^{m}
$$

where $m_{p}$ is the pay-load attached to the lqwer arm. It is to be preferred that the four matrices on the right hand side are mass independent. Since, it is the $M_{i}$ matrices, which are linearly dependent on $m_{p}$, this is however not the case. This leads to a pseudo-linear description where the four state space matrices in (6) depend on $m_{p}$. This might be seen as a problem, but the method proposed in this paper, is based on recursive estimation of $m_{p}$. That opens for the possibility of using the a priory estimate of $m_{p}$ in the expressions for the four state space matrices in (6).

We have in the previous sections established the following linear dependencies of $m_{p}$

$$
\begin{aligned}
& M_{2}=M_{2}^{0}+m_{p} M_{2}^{m} \\
& M_{3}=M_{3}^{0}+m_{p} M_{3}^{m} \\
& M_{5}=M_{5}^{0}+m_{p} M_{5}^{m}
\end{aligned}
$$

while $M_{1}, M_{4}, M_{6}$ and $M_{7}$ are independent of $m_{p}$. Notice (6) is not necessarily a Taylor expansion, but heavily rely on the definition of the four matrices in (6). If we define:

$$
\begin{aligned}
\underline{A}^{0}= & {\left[\begin{array}{ccc}
0 & M_{6} & 0 \\
0 & 0 & I \\
0 & \Lambda M_{1} & \Lambda M_{2}^{0}
\end{array}\right] \quad \underline{B}^{0}=\left[\begin{array}{c}
M_{7} \\
\Lambda M_{5} \\
\underline{B}_{3}^{0}
\end{array}\right] } \\
\underline{A}^{m}= & {\left[\begin{array}{ccc}
0 & 0 & 0 \\
0 & 0 & 0 \\
0 & 0 & \Lambda M_{2}^{m}
\end{array}\right] } \\
\underline{B}^{m}= & \left.\begin{array}{c}
0 \\
\Lambda M_{5}^{m} \\
\Lambda M_{2}^{m} \Lambda M_{5}^{0}+\Lambda M_{2}^{0} \Lambda M_{5}^{m}
\end{array}\right]
\end{aligned}
$$

where

$$
\underline{B}_{3}^{0}=\Lambda M_{2}^{0} \Lambda M_{5}^{0}+m_{p}^{2} \Lambda M_{2}^{m} \Lambda M_{5}^{m}+\Lambda M_{4}
$$

then (6) is fulfilled.

\section{The Measurement System}

The measurement system consists of two tachometers and four strain gauges. The tachometers give measurements of the link angles $\theta_{b 1}$ and $\theta_{2}$, whereas the strain gauges are located tactically on the links in order to give measurements of the deflections ie. $q$.

The measurement are connected to the state of the description through

$$
y_{t}=C x_{t} \quad C=\left[\begin{array}{ccc}
C_{t g} & 0 & 0 \\
0 & C_{s g 1} & 0 \\
0 & 0 & C_{s g 2}
\end{array}\right]
$$


where $C_{t g}, C_{s g 1}$ and $C_{s g 2}$ are observation matrices for the two tachometers and the strain gauges located on the two links. These are:

$$
\begin{gathered}
C_{t g}=\left[\begin{array}{cc}
k_{t g 1} & 0 \\
0 & k_{t g 2}
\end{array}\right] \\
C_{s g 1}=\left[\begin{array}{cc}
k_{s g 1} \varphi_{11}^{\prime \prime}\left(l_{11}\right) & k_{s g 1} \varphi_{12}^{\prime \prime}\left(l_{11}\right) \\
k_{s g 2} \varphi_{11}^{\prime \prime}\left(l_{12}\right) & k_{s g 2} \varphi_{12}^{\prime \prime}\left(l_{12}\right)
\end{array}\right]
\end{gathered}
$$

( $C_{s g 2}$ is defined in a similar manner). The constants $k_{t g i}$ and $k_{s g i}$ are constants characterizing the tachometers and the strain gauge, whereas $l_{j i}$ are the location (no $i$ ) on the the links (link no. $j$ ).

The end point location $\theta_{2 e}$ of the lower arm (second link) is given by:

$$
\begin{aligned}
\theta_{2 e}= & C_{m} x_{t} \\
C_{m}= & {\left[\begin{array}{ccc}
1 & 1 & \frac{\varphi_{11}(L)}{L_{1}} \ldots \frac{\varphi_{1 n}(L)}{L_{1}} \\
& \frac{\varphi_{21}(L)}{L_{2}} \ldots \frac{\varphi_{2 n}(L)}{L_{2}} & 0 \cdots 0
\end{array}\right] }
\end{aligned}
$$

\section{THE PAY-LOAD ESTIMATION}

So far the model of the dynamics has been studied in the continuous time form where the matrices in (6) exhibit a (pseudo) linear dependency on $m_{p}$. However, the discrete-time deltamodel shows (approximatively) a similar linearity of the pay-load as its continuous-time counterpart. This is due to the closed relation between the system matrices in continuous time and in the delta domain.

Let $z_{t}$ be a (eg. a vector) signal and $T$ the sampling interval, then the delta operator (cf. [4]) is defined by:

$$
\delta z_{t}=\frac{z_{t+T}-z_{t}}{T}
$$

One of its properties is related to the fact that a continuous time system

$$
\begin{aligned}
\dot{x} & =\underline{A} x_{t}+\underline{B} u_{t} \\
y_{t} & =\underline{C} x_{t}
\end{aligned}
$$

in discrete time is given by

$$
\begin{aligned}
\delta x_{t} & =A x_{t}+B u_{t} \\
y_{t} & =C x_{t}
\end{aligned}
$$

where

$$
A=\Omega \underline{A} \quad B=\Omega \underline{B} \quad C=\underline{C}
$$

and where

$$
\Omega=\frac{1}{T} \int_{0}^{T} e^{\underline{A} \tau} d \tau=I+\sum_{i=1}^{\infty} \frac{(\underline{A} T)^{i}}{(i+1) !}
$$

There exists a number of numerical procedures for determining the integral (and the infinite sum). Notice, if $T$ is chosen sufficient small then $A \simeq \underline{A}$ and $B \simeq \underline{B}$.

One of the interesting properties for the delta operator is that it preserve the mass split-up of state space matrices in (6). Let

$$
\begin{aligned}
A^{0} & =\Omega \underline{A}^{0} & B^{0}=\Omega \underline{B}^{0} \\
A^{m} & =\Omega \underline{A}^{m} & B^{m}=\Omega \underline{B}^{m}
\end{aligned}
$$

then the system is given by (12), where

$$
A=A^{0}+m_{p} A^{m} \quad B=B^{0}+m_{p} B^{m}
$$

One observes, that the suggested approach would not succeed in terms of a parameterization in the conventional shift-operator (since (13) does not hold for the shift operator version of $(12)$ ).

\section{A. Adaptation of the pay-load}

The system description in (12) is a deterministic description. When dealing with estimation and state estimation in particular one has to take effect of noise and disturbances into account. If a knowledge of the noise levels (variance) exists (or is obtained by estimation), then the states can be estimated by means of a Kalman filter. Other observer design can of course be applied. A predictive Kalman filter (with gain $K$ ) produces (see eg. [4]) an estimate of $x_{t+T}$ in terms of the filter

$$
\delta \hat{x}_{t}=A \hat{x}_{t}+B u_{t}+K\left(y_{t}-C \hat{x}_{t}\right)
$$

The estimate can be rephrased in a linear massdependent form

$$
\hat{x}_{t}=\hat{x}_{t}^{0}+m_{p} \hat{x}_{t}^{m}
$$

where

$$
\begin{aligned}
\hat{x}_{t}^{0}= & \left(I+T A^{0}-T K C\right) \hat{x}_{t-1} \\
& +T B^{0} u_{t-1}+T K y_{t-1} \\
\hat{x}_{t}^{m}= & T A^{m} \hat{x}_{t-1}+T B^{m} u_{t-1}
\end{aligned}
$$

The matrices, $A, B, C$ and $K$ are all calculated on the basis of information $\left(m_{p}\right)$ available at time $t-1$. Therefore all measurements are used to construct the best estimates of $\hat{x}_{t}$ and consequently also of $\hat{x}_{t}^{0}$ and $\hat{x}_{t}^{m}$.

Let us now focus on estimation of $m_{p}$. One method could be a variant of an extended Kalman filter in which both the states and the pay load mass are estimated. In this paper 
we will, however, use another method, which is based on a measurement of the end point position of the lower arm. This choice seems reasonable since the lower arm is mostly affected by variations in the pay-load. The method can of course be extended to include other measurements as well. With the sensor equipment mentioned in the introduction the end point position can not be measured directly, but have to be determined indirectly by means of the strain gauge and tachometer measurements.

Let $y_{t}^{m}$ denote the measurement of the end point position $\theta_{e 2}$. With $C_{m}$ from (10) we have the observation equation

$$
y_{t}^{m}=C_{m} x_{t}+e_{t}
$$

where $e_{t}$ is the inevitable measurement noise, which is assumed to be a zero mean white noise with variance equal $R_{2}$. This can also be expressed as:

$$
y_{t}^{m}=C_{m} \hat{x}_{t}+C_{m} \tilde{x}_{t}+e_{t}
$$

where the noise term $\varepsilon_{t}=C_{m} \tilde{x}_{t}+e_{t}$ is assumed to be zero-mean and uncorrelated with $\hat{x}_{t}$. That is the case if the design of a Kalman filter is based on correct data.

From (15) and (17) the least squares regression model

$$
\psi_{t}=\varphi_{t}^{\top} \theta_{t}+\varepsilon_{t}
$$

is produced with

$$
\begin{aligned}
\psi_{t}= & y_{t}^{m}-C_{m} \hat{x}_{t}^{0} \quad \varphi_{t}^{\top}=C_{m} \hat{x}_{t}^{m} \\
& \theta_{t}=m_{p}(t)
\end{aligned}
$$

The regression form is pseudo-linear since $\varphi_{t}$ is calculated on the basis of the estimate at time $t-1$.

To obtain a robust parameter identification a selective forgetting method is suggested, [6]. This algorithm shows tracking ability without the drawback of covariance blow-up. The method has a close parallel to the EFRA method and gains the same advantages at a less computational expense. This overcomes the problems of neither the covariance matrix tending to zero nor to blow-up. Also it guarantees that identification is always alert to some extent. The selective forgetting scheme (SF1) can be written as

$$
\begin{aligned}
\epsilon_{t} & =\psi_{t}-\varphi_{t}^{\top} \hat{\theta}_{t-1} \\
\kappa_{t} & =P_{t-1} \varphi_{t}\left[I+\varphi_{t}^{\top} P_{t-1} \varphi_{t}\right]^{-1}
\end{aligned}
$$

$$
\begin{aligned}
\hat{\theta}_{t} & =\hat{\theta}_{t-1}+\kappa_{t} \epsilon_{t} \\
P_{t \mid t-1} & =\frac{\alpha_{1}-\alpha_{0}}{\alpha_{1}} P_{t-1}+\alpha_{0} I \\
P_{t} & =\left(I-\kappa_{t} \varphi_{t}^{\top}\right) P_{t \mid t-1}
\end{aligned}
$$

where $0<\alpha_{0}<\alpha_{1}<\infty$. This algorithm (cf. $[6]$ ) is known to ensure that the eigen values of $P_{t}$ is bound between $\alpha_{0}$ and $\alpha_{1}$.

\section{Simulation EXPERIMENT}

In order to investigate the properties of the algorithm there have been performed a series of simulations. One simulation is described in the following.

The simulation (and the design) model of the deflection is a two mode approximation. The reference signals are square waves for the link angles and the signals are shown in Figure 3. The sampling period was chosen to $T=0.01 \mathrm{sec}$.
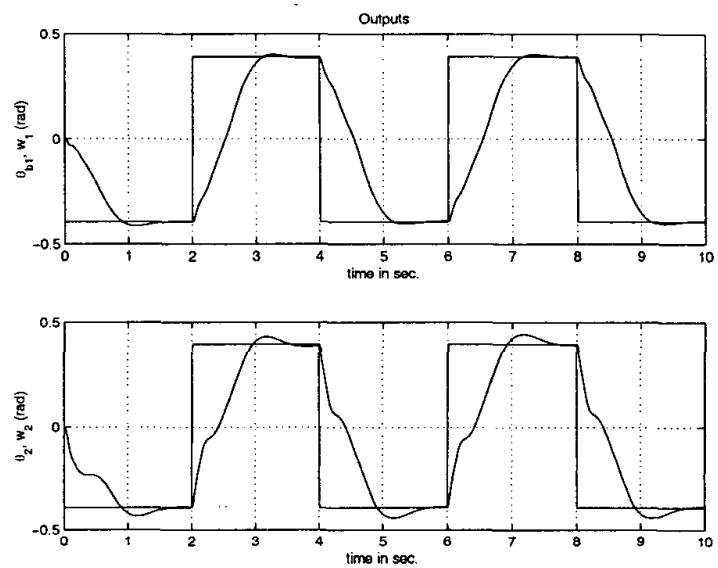

Fig. 3. Angular positions $\theta_{b 1}$ and $\theta_{2}$ presented as measured outputs (solid line) and as reference trajectories (dashed line).

figure

The controller is a state space LQG controller which parameters are iterated one step per sample in order to follow the adaptation on $m_{p}$. This would asymptotically producing the optimal feed-back gain based on the loss weights

$$
Q_{1}=C_{m}^{\top} C_{m}, \quad Q_{2}=0.005 I
$$

The control is based on the estimated state of the system which are obtained by means of the predictive Kalman filter with

$$
R_{1}=10^{-4} I \quad R_{2}=10^{-4} I
$$

where $R_{1}, R_{2}$ denote respectively the process and the measurement covariance matrices. In 
spite of the deterministic simulation, the process noise covariance is needed in order to compensate for the unknown pay load mass in the model.
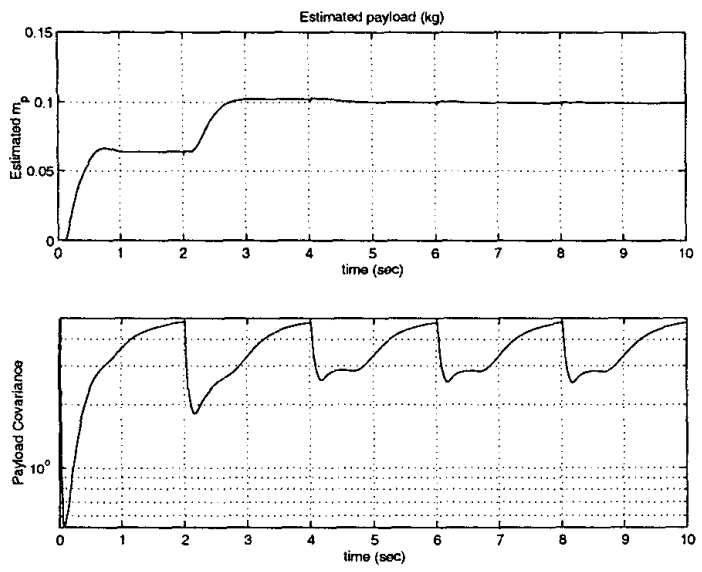

Fig. 4. Estimated pay-load $\hat{m}_{p}(t)$ and variance of estimate in a closed-loop experiment. figure

The payload mass was estimated using the following parameters

$$
\alpha_{0}=0.1 \quad \alpha_{1}=10 \quad P_{0}=10
$$

The value of $m_{p}=0.1 \mathrm{~kg}$ can be compared to e.g. the mass of the lower arm, $m_{l 2}=0.133 \mathrm{~kg}$, saying that the manipulator is heavily loaded. A better performance can be seen after the second step reflecting that $m_{p}$ is almost estimated after 3 seconds, see Figure 4 . From here it also appears that only when the set-points are varied new information is obtained.

\section{Conclusions}

The paper has presented a new method for online identification of pay-loads for a two-link flexible robot. First the state-space model has been derived, and then the model is discretised using the Delta-Operator, which benefits from the close correspondence between parameters of a discrete-time model and those of the underlying continuous-time model. Due to the close correspondence, it is shown that both domain models can produce almost the same linearity with respect to a pay-load. This fact is used in a pay-load estimation technique. By simulation it is demonstrated that it is possible to identify a time-varying pay-load of a two link flexible robot during closed-loop control.

\section{ACKNOWLEDGEMENT}

This research was gratefully supported by a grant from the Danish Research Council for Technical Science, STVF (Statens TekniskVidenskabelige Forskningsråd) under license no. 16-5059.

\section{REFERENCES}

[1] Jens Rane Baungaard. Modelling and Control Flexible Robot Links. PhD thesis, Department of Automation, The Technical University of Denmark, 1996.

[2] L. Kruise. Modelling and Control of a Flexible Beam and Robot Arm. PhD thesis, Universiteit Twente, 1990.

[3] A. D. Luca and S. Panzieri. An iterative scheme for learning gravity compensation in flexible robot arms. Automatica, 30(6):993-1002, 1994. Also as 12th World Congress of IFAC (1993), vol. 8, 191-198.

[4] R. Middleton and G. Goodwin. Digital Control and Estimation, - a Unified Approach. Prentice Hall, 1990.

[5] M. M'Saad, L. Dugard, and S. Hammad. A suitable generalized predictive adaptive controller case study of a flexible arm. Automatica, 29(3):589-608, 1993.

[6] J. E. Parkum. Recursive Identification of TimeVarying Systems. PhD thesis, IMSOR buil. 321, Technical University of Denmark, 1992. IMSOR No. $57 / 92$.

[7] M. Rostgaard. Modelling, Estimation and Control of Fast Sampled Dynamical Systems. PhD thesis, IMM buil. 321, Technical University of Denmark, 1995.

[8] Y. Sakawa, F. Matsuno, and S. Fukushima. Modelling and control of a flexible arm. Journal of Robotic Systems, 2:453-472, 1985. 